\section{PSICOLOGIA IBEROAMERICANA}

\section{Psicología Iberoamericana}

ISSN: 1405-0943

psicología.iberoamericana@uia.mx

Universidad Iberoamericana, Ciudad de

México

México

Nogueda Orozco, María José; Pérez Terán, Brenda; Barrientos Casarrubias, Vania; Robles García,

Rebeca; Sierra Madero, Juan G.

Escala de Ansiedad y Depresión Hospitalaria (HADS): validación en pacientes mexicanos con infección por VIH

Psicología Iberoamericana, vol. 21, núm. 2, julio-diciembre, 2013, pp. 29-37

Universidad Iberoamericana, Ciudad de México

Distrito Federal, México

Disponible en: http://www.redalyc.org/articulo.oa?id=133930525004

Cómo citar el artículo

- Número completo

- Más información del artículo

- Página de la revista en redalyc.org

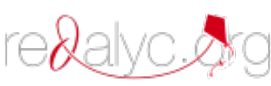

Sistema de Información Científica

Red de Revistas Científicas de América Latina, el Caribe, España y Portugal Proyecto académico sin fines de lucro, desarrollado bajo la iniciativa de acceso abierto 


\title{
Escala de Ansiedad y Depresión Hospitalaria (HADS): validación en pacientes mexicanos con infección por VIH
}

\author{
Hospital Anxiety and Depression Scale (HADS): validation in Mexican patients \\ with HIV infection
}

\author{
María José Nogueda Orozco ${ }^{1}$ \\ Brenda Pérez Terán \\ instituto Nacional de Ciencias Médicas y Nutrición \\ Vania Barrientos Casarrubias \\ Universidad Nacional Autónoma de México \\ Rebeca Robles García \\ Instituto Nacional de Psiquiatría \\ Juan G. Sierra Madero \\ instituto Nacional de Ciencias Médicas y Nutrición
}

\section{RESUMEN}

El HADs es un instrumento utilizado ampliamente para evaluar síntomas de ansiedad y depresión en el ámbito hospitalario. Se ha aplicado a pacientes con diversas enfermedades crónicas, principalmente cáncer y diabetes. El objetivo de este trabajo fue evaluar la confiabilidad y la validez del HADs en español, en pacientes mexicanos con diagnóstico de viн. Participaron 150 sujetos de ambos sexos con inicio reciente de tratamiento antirretroviral. El análisis factorial de componentes principales determinó un factor con valor eigen superior a uno que explica $54.65 \%$ de la varianza. El alfa de Cronbach para el total de la escala final de 13 reactivos fue de 0.90. Los reactivos de ansiedad y depresión estuvieron fuertemente correlacionados, lo que sugiere que el puntaje total puede medir distrés general. Es una escala breve y adecuada en tanto que descarta los síntomas somáticos propios de la enfermedad y del tratamiento antirretroviral.

Palabras clave: VIH, ansiedad, depresión, distrés psicológico, validación estadística.

\section{ABSTRACT}

HADS is an instrument that has been broadly used to evaluate symptoms of anxiety and depression in the hospital setting. It has been applied to patients with various chronic diseases, mainly cancer and diabetes. This article seeks to assess the reliability and validity of HADS in Spanish for Mexican patients diagnosed with HIV. The study involved 150 participants, of both sexes, who had recently begun antiretroviral therapy. The factor analysis of principal components determined a factor with an eigenvalue higher than one which explains $54.65 \%$ of the variance. Cronbach's alph a for the total of the final scale of 13 reagents was 0.90 . A strong correlation existed between anxiety and depression reagents, suggesting that the total score could measure general distress. It is a brief scale and one that is useful in discarding somatic symptoms that are an inherent part of the disease and antiretroviral therapy.

Keywords: HIV, anxiety, depression, psychological distress, statistical validation.

\footnotetext{
${ }^{1}$ Recibido: 19 de abril, 2013 - Aceptado: 24 de agosto, 2013

Correspondencia: María José Nogueda Orozco, Instituto Nacional de Ciencias Médicas y Nutrición Salvador Zubirán, Vasco de Quiroga 15, colonia Sección xvi, Tlalpan, México, D. F., C. P. 14000. Tel. 5487 09 00, ext. 5506. mj_nogueda@hotmail.com
} 


\section{INTRODUCCIÓN}

La Escala de Ansiedad y Depresión Hospitalaria (HADS, por sus siglas en inglés) se ha utilizado ampliamente para evaluar el malestar emocional en pacientes con diferentes padecimientos crónicos. Fue creada por Zigmund y Snaith (1983) para la evaluación de los síntomas cognitivos y conductuales y no así los síntomas somáticos de la ansiedad y la depresión como insomnio, fatiga, pérdida y/o aumento de peso y/o apetito. Ha sido aplicada en pacientes que acuden con regularidad a ambientes hospitalarios, no psiquiátricos, con lo cual resulta una mejor herramienta para la detección de distrés psicológico en población médicamente enferma (en comparación con el resto de escalas de autoaplicación en el área) y evitar equívocos de atribución cuando se aplica a individuos con algún tipo de enfermedad.

Desde su publicación, el HADs ha sido traducido a más de 25 idiomas y varias poblaciones (Hermann, 1977). La traducción al español y su validación fue realizada por Tejero, Guimerá, Farré \& Peri (1986). La reciente revisión de Bjelland, Dahl, Haug y Neckelmann (2002) reportó que el HADs ha demostrado altos niveles de confiabilidad y validez en su uso con pacientes en varias condiciones médicas. Específicamente en México, se ha validado en cáncer de mama y obesidad (Ornelas-Mejorada, Tufiño, \& Sánchez-Sosa, 2011; López-Alveranga et al., 2002) demostrando adecuadas propiedades psicométricas.

Entre los diversos factores emocionales relacionados con el Vir destacan la ansiedad y la depresión. Se ha reportado que la prevalencia de estos trastornos es mayor en población con viH (Berg, Michelson \& Safren, 2007). De la misma manera, se ha resaltado la importancia del mantenimiento de un buen estado de ánimo que no limite la adherencia al tratamiento y permita un adecuado ajuste a la enfermedad. Así, la detección de estos padecimientos adquiere mayor relevancia (Fernández \& Ruiz, 2006; Nogueira, De Fátima \& Guimarães, 2006).

En el caso de la infección por viH, algunas manifestaciones físicas, o incluso los efectos adversos del tratamiento antirretroviral, pueden llegar a confundirse con síntomas somáticos de la ansiedad y la depresión, ocasionando un subdiagnóstico de estos padecimientos (Asch et al., 2003); por eso, el HADs podría ser una herramienta idónea para la identificación oportuna de distrés psicológico en esta población.

Además, la evaluación de la constelación particular de síntomas depresivos y ansiosos en casos de viH permitirá la generación de evidencia no sólo de la utilidad del HADS en nuestro contexto y en este tipo de pacientes, sino de la manera en que se manifiesta el distrés psicológico que aqueja a personas médicamente enfermas en general.

En estudios previos, el análisis factorial del HADS comúnmente indica dos dimensiones sugeridas por los autores (Snaith, 2003). Sin embargo, en algunos estudios se han encontrado más de dos dimensiones (Watson et al., 1995; Savard, Laberge, Gauthier, Ivers \& Bergeron, 1998; Colin, 2005) o una sola (Razavi, Delvaux, Favacques \& Robay, 1990; Reda, 2011), lo que sugiere que el comportamiento de la escala y los constructos que evalúa son diferentes según la población clínica a la que se aplique.

En esta dirección, el objetivo del presente estudio fue probar las propiedades psicométricas del HADs en pacientes mexicanos que viven con viH.

\section{MÉTODO}

\section{Instrumento}

El HADS es un cuestionario de autoaplicación de 14 reactivos, integrado por dos subescalas de siete reactivos cada una, una de ansiedad (reactivos impares) y otra de depresión (reactivos pares).

Los autores de la versión original en inglés (Zigmund \& Snaith, 1983) se propusieron, desde el inicio del proceso de desarrollo del instrumento, definir cuidadosamente y distinguir claramente entre los conceptos de ansiedad y depresión. Los ocho reactivos de la subescala de ansiedad están seleccionados a partir del análisis y la revisión de la escala Present State Examination (PSE), así como de la investigación acerca de las manifestaciones psíquicas de la ansiedad (Zigmund \& Snaith, 1983). De esta manera, los reactivos se dirigen a conocer si la persona ha estado tensa, preocupada o ha tenido sensaciones de temor. Por su parte, los ocho reactivos de la subescala de depresión se centran en el área de la anhedonia, puesto que es probablemente el rasgo psicopatológico central de la depresión (Zigmund \& Snaith, 1983). Así, la subescala 
de depresión indaga, básicamente, cuestiones como la pérdida de interés en las actividades cotdianas, tener pensamientos pesimistas o la capacidad de reír.

El tiempo total de respuesta es de aproximadamente diez minutos. En las instrucciones se pide al paciente que describa cómo se ha sentido durante la última semana incluyendo el día de hoy.

Las opciones de respuesta son tipo Likert que oscilan del cero al tres, dando un puntaje mínimo de cero y un puntaje máximo de 21 para cada subescala. Originalmente, los puntos de corte de cero a siete implican la ausencia de ansiedad y/o depresión clínicamente relevante, de ocho a diez que requiere consideración y del 11 al 21 la presencia de sintomatología relevante y un probable caso de ansiedad y/o depresión (Snaith, 2003).

\section{Participantes}

Con base en un muestreo por conveniencia (Carretero-Dios \& Pérez, 2005) se incluyeron a todos los pacientes mayores de dieciocho años que iniciaron tratamiento antirretroviral en el periodo de enero de 2010 a agosto 2011, en la Clínica de viH del Instituto Nacional de Ciencias Médicas y Nutrición Salvador Zubirán (INCMNSz) y que aceptaron participar en el estudio de manera confidencial y voluntaria.

\section{Procedimiento}

Dos psicólogas del servicio de Infectología del INCMNSz estuvieron encargadas de invitar a los pacientes a participar en un protocolo sobre "Factores psicosociales asociados a inicio tardío de tratamiento antirretroviral" (aprobado por el Comité de Ética del InNsz, Ref. 172) que consistía en contestar una serie de cuestionarios entre los que se incluía el HADs. También se evaluaron variables sociodemográficas y clínicas como edad, sexo y conteo de CD4.

\section{Análisis de datos}

Para las variables sociodemográficas y clínicas se realizó un análisis descriptivo con media, desviación estándar y rango para las continuas, así como frecuencias y porcentajes para las categóricas. Se determinó la correlación Pearson entre las subescalas y la escala total.
Para la validación del cuestionario, en primer lugar se realizó un análisis de frecuencia para cada uno de los reactivos, con el fin de verificar su capacidad de discriminación y la adecuación de las opciones de respuesta. Después se calcularon los puntajes totales y subtotales para cada participante.

La validez del constructo se evaluó con base en un análisis factorial de componentes principales con rotación varimax. La validez interna se determinó con el coeficiente de correlación intraclase y la confiabilidad de la prueba se calculó con alfa de Cronbach.

\section{RESULTADOS}

Se incluyeron 150 sujetos, de los cuales 15 (11.4\%) fueron del sexo femenino y 135 (88.6\%) del sexo masculino. En la tabla 1 se muestran las principales características sociodemográficas y clínicas de la muestra.

Las puntuaciones medias, en el HADS versión español para México, fueron de $7.61( \pm 3.98)$ para ansiedad y de 4.89 ( \pm 4.39$)$ para depresión, en un rango de cero a 21 para ambas; el puntaje total promedio de la muestra fue de $12.5( \pm 8.37)$, en un rango de cero a 42 . La correlación entre las subescalas de ansiedad y depresión fue de 0.77 ( $\mathrm{p} \leq 0.001$ ), mientras que la correlación entre la subescala de ansiedad y la escala total fue de 0.92 ( $\mathrm{p} \leq 0.001$ ); para la subescala de depresión y la escala total fue de 0.93 ( $\mathrm{p} \leq 0.001)$. 
Tabla 1. Características sociodemográficas y clínicas de la muestra

\begin{tabular}{|c|c|c|}
\hline \multicolumn{2}{|c|}{ Total de participantes $\mathrm{N}=150(100 \%)$} & n (\%) \\
\hline Sexo & $\begin{array}{l}\text { Femenino } \\
\text { Masculino }\end{array}$ & $\begin{array}{r}15(10) \\
135(90)\end{array}$ \\
\hline Edad (años) & $\begin{array}{l}\text { Grupo } 1>34 \\
\text { Grupo } 2 \geq 34 \text { y }<45 \\
\text { Grupo } 3 \geq 45\end{array}$ & $\begin{array}{l}74(49.33) \\
49(32.67) \\
27(18)\end{array}$ \\
\hline Educación (años) & $\begin{array}{l}<9 \\
>9\end{array}$ & $\begin{array}{r}39(26) \\
111(74)\end{array}$ \\
\hline Pareja estable & $\begin{array}{l}\text { Sí } \\
\text { No }\end{array}$ & $\begin{array}{l}72(48) \\
78(52)\end{array}$ \\
\hline Nivel socioeconómico & $\begin{array}{l}\text { Bajo }^{\mathrm{a}} \\
\text { Medio-Alto }\end{array}$ & $\begin{array}{r}40(26.66) \\
110(73.33)\end{array}$ \\
\hline Ocupación & $\begin{array}{l}\text { Sin empleo } \\
\text { Con empleo }\end{array}$ & $\begin{array}{l}61(40.66) \\
89(59.33)\end{array}$ \\
\hline Riesgo de transmisión & $\begin{array}{l}\mathrm{HSH}^{*} / \text { bisexual } \\
\text { Heterosexual y otros }\end{array}$ & $\begin{array}{l}95(63.33) \\
55(36.66)\end{array}$ \\
\hline
\end{tabular}

${ }^{*} \mathrm{HSH}=$ hombres que tienen sexo con hombres.

${ }^{a}$ Nivel bajo comprende del uno y dos y nivel medio-alto comprende del tres al siete, según la clasificación socioeconómica que realiza el InNsz.

En la tabla 2 se muestran las correlaciones de cada reactivo con el total de las subescalas.

El alfa de Cronbach para la subescala de ansiedad fue de 0.78 y para la subescala de depresión de 0.85 . Los coeficientes de correlación intraclase fueron $78.2 \%$ (IC $95 \%$ 72.5-83.2, $\mathrm{p}=0.00$ ) y $87.7 \%$ (IC 95\% 84.4-90.5, $\mathrm{p}=0.00)$ para ansiedad y depresión, respectivamente. El índice de adecuación muestral de Kayser-MeyerOlkin fue de 0.90. El análisis de componentes rotados reveló un factor principal (11 reactivos) y un indicador (dos reactivos) que explican $54.65 \%$ de la varianza (tabla 3). La confiabilidad no aumentaba ni disminuía al eliminar los dos reactivos del indicador, por lo que se decidió conservarlos. El reactivo que se eliminó fue el seis (de depresión) por tener cargas factoriales similares en ambos factores.
La escala final quedó compuesta por 13 reactivos (ver anexo). Para esta nueva versión se tiene un puntaje mínimo de cero y máximo de 39 . Se sugiere un punto de corte de cero al nueve para ausencia de distrés, diez a 19 presencia de distrés leve, 20 a 29 moderado y 30 a 39 grave.

Con los puntos de corte originales del cuestionario se encontró una prevalencia de ansiedad de 56\% y de depresión de $33 \%$ en el total de la muestra. Con los puntos de corte sugeridos en este estudio se halló una prevalencia de $47.3 \%$ en ausencia de distrés, $36.7 \%$ leve, $23 \%$ moderado y $7 \%$ grave. Se pueden ver similitudes en las prevalencias de ansiedad y depresión si se suman las categorías de distrés. 
Tabla 2. Correlación de reactivos con el total de cada subescala

\begin{tabular}{|c|c|c|}
\hline Reactivos & HADS-A & HADS-D \\
\hline Ansiedad & 1 & \\
\hline 1. Me siento tenso o nervioso & 0.697 & 0.607 \\
\hline 3. Siento una especie de temor como si algo malo fuera a suceder & 0.706 & 0.506 \\
\hline 5. Tengo la cabeza llena de preocupaciones & 0.690 & 0.602 \\
\hline 7. Soy capaz de permanecer sentado, tranquilo y relajado & 0.582 & 0.418 \\
\hline 9. Experimento una desagradable sensación de "nervios y hormigueos" en el estómago & 0.650 & 0.492 \\
\hline 11. Me siento inquieto como si no pudiera parar de moverme & 0.479 & 0.359 \\
\hline 13. Experimento de repente sensaciones de gran angustia o temor & 0.810 & 0.610 \\
\hline Depresión & & 1 \\
\hline 2. Sigo disfrutando de las cosas como siempre & 0.681 & 0.757 \\
\hline 4. Soy capaz de reírme y ver el lado gracioso de las cosas & 0.602 & 0.785 \\
\hline 6. Me siento alegre & 0.647 & 0.780 \\
\hline 8. Me siento lento y torpe & 0.467 & 0.698 \\
\hline 10. He perdido el interés por mi aspecto personal & 0.579 & 0.750 \\
\hline 12. Espero las cosas con ilusión & 0.565 & 0.793 \\
\hline 14. Soy capaz de disfrutar con un buen libro o con un buen programa de radio o televisión & 0.544 & 0.751 \\
\hline
\end{tabular}


Tabla 3. Matriz de componentes rotados y cargas factoriales de los reactivos

\begin{tabular}{|c|c|c|}
\hline Reactivos & Factor & Indicador \\
\hline 1. Me siento tenso o nervioso & 0.808 & \\
\hline 2. Sigo disfrutando de las cosas como siempre & 0.679 & \\
\hline 3. Siento una especie de temor como si algo malo fuera a suceder & 0.593 & \\
\hline 4. Soy capaz de reírme y ver el lado gracioso de las cosas & 0.736 & \\
\hline 5. Tengo la cabeza llena de preocupaciones & 0.766 & \\
\hline 6. Me siento alegre & 0.584 & 0.546 \\
\hline 7. Soy capaz de permanecer sentado, tranquilo y relajado & & 0.750 \\
\hline 8. Me siento lento y torpe & 0.653 & \\
\hline 9. Experimento una desagradable sensación de "nervios y hormigueos" en el estómago & 0.532 & \\
\hline 10. He perdido el interés por mi aspecto personal & 0.658 & \\
\hline 11. Me siento inquieto como si no pudiera parar de moverme & & 0.818 \\
\hline 12. Espero las cosas con ilusión & 0.642 & \\
\hline 13. Experimento de repente sensaciones de gran angustia o temor & 0.688 & \\
\hline 14. Soy capaz de disfrutar con un buen libro o con un buen programa de radio o tele & 0.635 & \\
\hline
\end{tabular}

\section{DISCUSIÓN}

Los datos de este estudio demuestran que el HADs es una herramienta de fácil aplicación y de utilidad para detectar casos de ansiedad y depresión en población con vir. La versión en español sujeta a estudio se caracterizó por una adecuada consistencia interna. Las subescalas correlacionaron de manera importante entre sí, lo cual concuerda con lo reportado previamente en la literatura: la ansiedad y la depresión son diagnós- ticos que suelen estar interrelacionados (Hermann, 1997; Bjelland et al., 2002). Se puede observar cierta tendencia a presentar mayores coeficientes de correlación en la dimensión a la que corresponden los reactivos.

Así, aunque la estructura factorial del instrumento no coincidió con la de dos factores reportada para la versión original de Zigmund y Snaith (1983); se confirmó la existencia de un factor de distrés general como 
en los estudios previos de Razavi et al. (1990) y Reda (2011), realizados en población con VIH de Francia y Etiopía, respectivamente.

Tal como sugieren Watson et al. (1995), la fenomenología de la ansiedad y la depresión es claramente diferente; la primera está centrada en la emoción de miedo e involucra sentimientos de preocupación, en contraste con la segunda que está dominada por la emoción de tristeza, y se asocia con sentimientos de desesperanza; sin embargo, es difícil distinguir de manera empírica ambos constructos, que aparecen íntimamente correlacionados en las medidas de autorreporte; esto parece ser cierto, en particular, en pacientes con VIH.

$\mathrm{Al}$ encontrar que los reactivos de ansiedad y depresión estuvieron fuertemente correlacionados y se agruparon en un factor, se propone que el puntaje total del HADs evalúa de forma global el distrés psicológico, en lugar de depresión y ansiedad como entidades separadas y diferenciadas. Este único factor encontrando (distrés psicológico) puede definirse como un estado emocional de sufrimiento caracterizado por síntomas ansiosos y depresivos (Mirowsky \& Ross, 2002). El constructo se ha sugerido como un indicador de la salud mental en estudios de salud pública y como desenlace de intervenciones clínicas (Drapeau, Marchard \& Beaulieu-Prèvost, 2012).

Además, se pudo constatar una alta prevalencia de síntomas de ansiedad y depresión o distrés psicológico en pacientes mexicanos con viH; esto es congruente con la literatura previa, por lo que se sugiere

\section{REFERENCIAS}

Asch, S., Kilbourme, A., Gifford, A., Burnman, M., Turner, B. Shapiro, M., et al. (2003). Underdiagnosis of depression in HIV: who are we missing? Journal of General Internal Medicine, 18(6), 450-460.

Berg, C., Michelson, S. \& Safren, S. (2007). Behavioral aspects of HIV care: adherence, depression, substance use, and HIv-transmission behaviors. Infectious Diseases Clinics of North America, 21(1), 181-200.

Bjelland, I., Dahl, A., Haug, T. \& Neckelmann, D. (2002). The validity of the Hospital Anxiety and Depression Scale. An updated literature review. Journal of Psychosomatic Research, 52, 69-77. el HADS como un parámetro clínico que puede ser de gran utilidad en la detección temprana y el tratamiento de esta problemática y sus complicaciones.

El HADs evalúa los síntomas no somáticos del distrés psicológico; esto representa una gran ventaja en comparación con otras medidas de evaluación, ya que en ocasiones estos síntomas pueden atribuirse a la enfermedad o a los efectos adversos del tratamiento. Además, es una medida con buena aceptación en su aplicación, pues el tiempo de respuesta es breve y su redacción es de fácil comprensión.

Entre las limitaciones de este estudio destacan que, al tratarse de una investigación en proceso, con una muestra no representativa de la población, los datos de frecuencia de distrés (ansiedad y depresión) no pueden ser generalizados a toda la población. Más aún, en relación con el objetivo central del estudio, la muestra incluyó a pacientes en su mayoría hombres, con escolaridad superior a nueve años, y en nivel socioeconómico medio-alto, con lo que es necesario evaluar si la escala resulta también adecuada para pacientes con características demográficas diferentes.

Finalmente, se sugiere que futuras investigaciones se dirijan a la evaluación de la sensibilidad al cambio de la escala, con base en su aplicación repetida en las subsecuentes visitas de los pacientes. Esto permitirá conocer si además es una herramienta valiosa para determinar la efectividad de las intervenciones terapéuticas, a fin de disminuir el distrés que tan frecuentemente afecta a esta población. •

Carretero-Dios, H. \& Pérez, C. (2005). Normas para el desarrollo y revisión de estudios instrumentales. International Journal of Clinical and Health Psychology, 5, 521-551.

Colin, M. (2005). What does the Hospital Anxiety and Depression Scale (HADs) really measure in liaison psychiatry settings? Current Psychiatry Reviews, 1(1), 69-73.

Drapeau, A., Marchand, A. \& Beaulieu-Prévost, D. (2012). Epidemiology of psychological distress, mental illnesses - understanding, prediction and control. Labate, L. (Ed.) ISBN: 978-953-307-662-1, InTech. 
Disponible en: http://www.intechopen.com/books/ mental-illnesses-understanding-prediction-andcontrol/epidemiology-of-psychological-distress

Fernández, F. \& Ruiz, P. (2006). Psychiatric aspects of HIV/ AIDS. Philadelphia: Lippincot Williams and Wilkins.

Hermann, C. (1997). International experiences with the hospital anxiety and depression scale - a review of validation data and clinical results. Journal of Psychosomatic Research, 42, 17-41.

López-Alveranga, J., Vázquez, V., Arcila, D., Sierra, A., González, J. \& Salín, R. (2002). Hospital Anxiety and Depression Scale (HAD) en sujetos obesos mexicanos. Revista de Investigación Clínica, 54 (5), 403-409.

Mirowsky, J. \& Ross, C. (2002). Selecting outcomes for the sociology of mental health: issues of measurement and dimensionality. Journal of Health and Social Behavior, 43, 152-170.

Nogueira, L., De Fátima, P. \& Guimarães, M. (2006). Anxiety and depression assessment prior to initiating antiretroviral treatment in Brazil. AIDS care, 18(6), 529-536. doi:10.1080/09540120500221704

Ornelas-Mejorada, R., Tufiño, M. \& Sánchez-Sosa, J.J. (2011). Ansiedad y depresión en mujeres con cáncer de mama en radioterapia: prevalencia y factores asociados. Acta de Investigación Psicológica, 1(3), 401-414.

Razavi, D., Delvaux, N., Farvacques, C. \& Robay, E. (1990). Screening for adjustment disorders and ma- jor depressive disorders in cancer in-patients. British Journal of Psychiatry, 156, 79-83.

Reda, A. (2011). Reliability and validity of the ethiopian version of the Hospital Anxiety and Depression Scale (HADS) in HIV infected patients. PLoS ONE, 6(1), e16049. doi:10.1371/journal.pone.0016049

Savard, J., Laberge, B., Gauthier, J., Ivers, H. \& Bergeron, M. (1998). Evaluating anxiety and depression in HIVinfected patients. Journal of Personality Assesment, 71, 349-367.

Snaith, R. (2003). The hospital anxiety and depression scale. Health and Quality of Life Outcomes, I, 29.

Tejero, A., Guimerá, E., Farré, J. \& Peri, J. (1986). Uso clínico del HAD (Hospital Anxiety and Depression Scale) en población psiquiátrica: un estudio de sensibilidad, fiabilidad y validez. Revista del Departamento de Psiquiatría de la Facultad de Medicina de Barcelona, 12, 233-238.

Watson, D., Weber, K., Assenheimer, J., Clark, L., Strauss, M \& McCormick, R. (1995). Testing a tripartite model: evaluating the convergent and discriminant validity of anxiety and depression symptom scales. Journal of Abnormal Psychology, 104, 3-14.

Zigmund, A. \& Snaith, R. (1983). The hospital anxiety and depression scale. Acta Psiquiátrica Escandinava, $67,361-370$. 


\section{ANEXO}

HADS-VIH

Instrucciones: A continuación se presenta una lista de afirmaciones que describen una serie de estados emocionales. Lea cada pregunta y marque con una $\mathrm{X}$ la respuesta que mejor indique cómo se ha sentido en esta última semana incluyendo el día de hoy.

1. Me siento tenso(a) o nervioso(a):

Todo el día Gran parte del día

De vez en cuando

Nunca

2. Sigo disfrutando de las cosas como siempre:

Igual que antes No tanto como antes Solamente un poco Ya no disfruto como antes

3. Siento una especie de temor como si algo malo fuera a suceder:

\begin{tabular}{l|l|l|l|l} 
Sí y muy intenso & Sí, pero no muy intenso & Sí, pero no me preocupa & No siento nada de eso
\end{tabular}

4. Soy capaz de reírme y ver el lado gracioso de las cosas:

Igual que siempre Actualmente, algo menos

Actualmente, mucho menos

Actualmente, nada

5. Tengo la cabeza llena de preocupaciones:

\begin{tabular}{l|l|l|l} 
Todo el día & Gran parte del día & De vez en cuando & Nunca
\end{tabular}

6. Me siento lento(a) y torpe:

Todo el día

Gran parte del día

De vez en cuando

Nunca

7. Soy capaz de permanecer sentado(a) tranquilo(a) y relajado(a):

\begin{tabular}{l|l|l} 
Siempre & A menudo & Raras veces
\end{tabular}

8. He perdido el interés por mi aspecto personal:

\begin{tabular}{l|l|l|l} 
Completamente & A menudo & Rara vez & Nada
\end{tabular}

9. Experimento una desagradable sensación de "nervios y hormigueos" en el estómago:

\begin{tabular}{l|l|l|l} 
Siempre & A menudo & Rara vez & Nunca
\end{tabular}

10. Espero las cosas con ilusión:

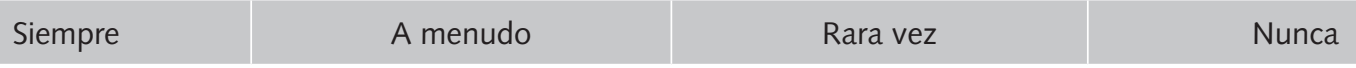

11. Me siento inquieto(a) como si no pudiera parar de moverme:

\begin{tabular}{l|l|l|l} 
Siempre & A menudo & Rara vez & Nunca
\end{tabular}

12. Soy capaz de disfrutar con un buen libro o con un buen programa de radio o televisión:

\begin{tabular}{c|c|c|c} 
Siempre & A menudo & Rara vez & Nunca
\end{tabular}

13. Experimento de repente sensaciones de gran angustia o temor:

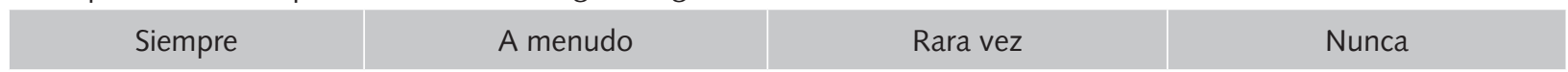

